While reading this book I was on duty for the A\&E department and looked up $\frac{\mathbb{\Phi}}{0}$ some of the more unusual conditions that presented that day. Brodie's abscess 3 was not listed in the index and the only reference to osteomyelitis in children was $\stackrel{\mathbb{Q}}{\stackrel{0}{\circ}}$

one paragraph in a book of over a thousand pages.
Fracture/dislocation of the ankle with an obvious threat to the viability of the overlying skin was not covered. There is an algorithm for treating fractures but no mention is made of the importance of preserving circulation to the skin in severe dislocations.

The book generally covers the unusual, such as, environmental emergencies quite well but day to day emergencies suffer as they are given equal coverage to $\vec{O}$ the more exotic.

In summary this is an interesting book that is well-produced and contains some $\vec{\omega}$ very well prepared and informative pieces. Overall, however, it does not provide $\frac{\mathbb{\Phi}}{3}$ enough information on the more common emergencies for it to be of daily use in an A\&E department. Furthermore, it is written entirely for the North American ${ }_{\omega}^{\circ}$ market and many of its recommendations are clearly not applicable to British Practice.

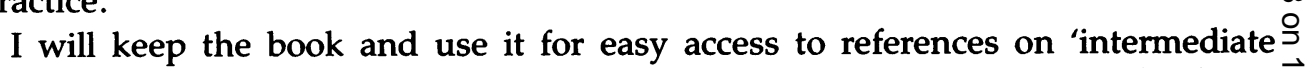
exotica'. It will not provide the department with an easily useable reference book nor $\vec{\omega}$ provide myself with a definitive text on the rarer conditions that we occasionally see. $\frac{\mathbb{D}}{\mathbb{D}}$

A. D. REDMOND

N. Staffordshire Trauma Centre

Stoke-on-Trent

Staffordshire

\title{
Poisoning and Drug Overdose
}

By K. Olson

Large \& Appleton, Hemel Hempstead. 1990, £10.95.

This book is aptly described by the Publishers as a 'clinical manual'. It is an excellent source of information for the emergency physician and is divided into four sections.

The first section details methodology and rationale for urgent evaluation of a 3 . poisoned patient, using the airway, breathing and circulation approach. It offers a $\frac{\sigma}{3}$. number of lists of clinical symptoms, supported by the drugs which may induce them, and then describes the appropriate laboratory investigations to confirm clinical suspicion into firm diagnosis. Methods of eliminations are also discussed.을

The second section lists a number of specific drugs and poisons in alphabetical $\frac{D}{2}$ order. A description follows of the availability of each compound mechanism of toxicity, clinical presentation, diagnosis and treatment. At least one reference is $N$ given for each agent as supplementary reading. The list of poisons also includes $N$ plants and snake and spider bites.

The third section lists specific antidotes to previously mentioned toxins and describes when and how they should be used.

Section four is named Environmental and Occupational Toxicology. There is a brief description of the theory of medical management in relation to a major ${ }_{0}^{-}$ 
incident involving toxic chemicals. The long table of health hazard summaries for industrial and occupational chemicals may well be useful under such circumstances, as may the list of suspected human carcinogens. These have less relevance for the practising Accident and Emergency (A\&E) physician but this section is brief and worth keeping within the clinical manual as a reference source.

The only small disappointment was that a book described as the International Edition' should detail lists of poisons centres and methods of labelling toxic compounds during transport, relating only to the United States. However, despite that, it is an extremely useful and easily read, well set out source of information that should be in every A\&E department and probably in the pocket of anyone involved in the emergency management of a poisoned patient.

\section{WATERS}

Stockport Infirmary

Stockport

Cheshire

\section{Practical Fracture Treatment (2nd Edition)}

By R. MCRAE

Churchill Livingstone, Edinburgh. 1989, £22.95, 328pp.

The successful format of annotated diagrams and self-test questions and answers has been reproduced and extended for the second edition of this very useful book.

The first part is suitable for under- or post-graduate students with a minimal prior knowledge of orthopaedics. It sets out the basic pathology of fractures, principles of diagnosis and management, and recognition of complications.

The second, and longer part, considers bony injuries region by region. Of particular value to the accident and emergency (A\&E) doctor and aspiring orthopaedic surgeon is the emphasis that has been placed on the mechanism by which injury may be produced. Line drawings convey the effects of applying percussive and rotational forces to single bones and joints and more complex 'ring' structures. The quality of reproduction of $X$-rays to illustrate this is excellent. Drawings are then used to demonstrate the method of reduction for displaced fractures. There are a considerable number of self-test questions which reinforce earlier teaching.

The author stresses in the preface that he does not seek to be dogmatic with respect to treatment, but to present a range of accepted treatment options.

Practical Fracture Treatment is successful not only as a quick reference guide for the junior doctor, but also as a manual which explains how the bony skeleton sustains and responds to injury. It should be in every A\&E department and on the bookshelves of anyone involved with treating injured patients.

M. WATERS

Stockport Infirmary

Stockport

Cheshire 